improvement, including the timing of course and coverage of the curriculum. To date, six medical officers are pursuing this pathway with three of them passing one paper and another two pursuing the final part.

Conclusion. The Northern STARS project is an ecosystem of training solutions while generating income and producing more local talents to expand this project further. More long-term evaluation from the perspective of human resource and health economics can be considered to understand the efficiency of the current initiative.

\section{Peer mentoring in psychiatry: a trainee-led initiative}

Zoe Moore $^{1 \star}$, Linda Irwin ${ }^{2}$, Stuart Brown ${ }^{1}$, Julie Anderson ${ }^{2}$ and Stephen Moore ${ }^{3}$

${ }^{1}$ Belfast Health and Social CareTrust; ${ }^{2}$ Northern Health and Social Care Trust and ${ }^{3}$ South Eastern Health and Social Care Trust ${ }^{*}$ Corresponding author.

doi: 10.1192/bjo.2021.418

Aims. Our aim was to establish a Peer Mentoring Network within Psychiatry Training in Northern Ireland.

Recognising that starting a new job can be a stressful time in any junior doctor's career, we wanted to ensure that new Core Trainees (CT1s) joining our Specialty Programme were well supported through this transition.

Although Clinical and Educational Supervision is well established in providing a support structure for trainees, we believed that a peer mentoring relationship, (with allocation of a Higher Psychiatry Trainee as mentor), would be of additional benefit.

It was hoped that the scheme would prove mutually beneficial to both mentee and mentor.

Method. We delivered a presentation at CT1 induction and sent out follow-up emails to encourage participation. Higher trainees were also sent information via email and asked to complete a basic application form if interested in becoming a mentor. Prospective mentors then attended a one-day training session.

Two lead mentors, (also higher trainees), were allocated to oversee the scheme, with additional supervision from two lead Consultants. Mentor-Mentee matches were made based on information such as location, sub-specialty affiliations and outside interests.

Matched pairs were advised about the intended frequency and nature of contacts. Check-in emails were sent halfway through the year and feedback evaluations completed at the end.

Result. 95\% of trainees who completed the evaluations said they would recommend the scheme to colleagues.

Mentees reported benefits in terms of personal and professional development, whilst mentors reported improved listening, coaching, and supervisory skills.

A small number of trainees highlighted that 6 monthly rotations impacted on ability to maintain face to face contacts.

Recruitment and engagement have improved annually. We are currently running the third year of the scheme and have achieved $100 \%$ uptake amongst CT1s and are over-subscribed with mentors, (19 mentors to 13 mentees).

Conclusion. The majority of feedback received has been positive and interest in the scheme continues to grow.

Potential issues relating to location of postings has been overcome, at least in part, by recent changes to ways of working and the use of alternative forms of contact, such as video calling.

Having exceeded demand in terms of mentor recruitment, we hope to extend the scheme to include trainees of other grades, and particularly those who are new to Northern Ireland.
We are excited to see where the next stage of our journey takes us and hope that others will be inspired to embark on similar schemes within their areas of work.

Quantitative and qualitative analysis of feedback from The Psychiatry Teaching Programme for Foundation Year doctors rotating through Pennine acute trust from 2010 to 2020

Angel Namuddu ${ }^{1 *}$, Margaret Gani ${ }^{2}$ and Sarah Burlinson ${ }^{2}$

${ }^{1}$ Manchester University NHS Foundation Trust and ${ }^{2}$ Search Results Pennine Care NHS Foundation Trust

${ }^{*}$ Corresponding author.

doi: 10.1192/bjo.2021.419

Aims. To monitor the year on year trend of feedback scores regarding content, presentation and relevance of sessions delivered as part of the programme by analysing the average Likert scales. To review the confidence post topic from FY feedback. To review qualitative data on the written feedback annually using a word cloud.

Method. Collated data from teaching programme from the various teaching sessions from the past decade and analysed previous teaching reports completed by previous ST leads.

Result. Finding: Relevance: Improvement in the average score year on year, highest in 2018/19 at 4.8/5

Content: Improvement in the average score year on year, highest in 2018/19 at 4.6/5.

Delivery: Improvement in the average score year on year, highest in 2018/19 at 4.6/5.

Qualitative analysis showed that the common themes that were commented on as positives for the session were: interactive, relevant and interesting, for areas for improvements the common themes were: more interaction, split into shorter sessions, faster pace and the need practical advice

Conclusion. Recommendations: teaching for FYs should aim to be interactive, relevant and interesting and include practical advice, be shorter and faster paced. Teaching programme organisers to contine to use the foundation year feedback to improve the teaching programme including advising future trainees and organising different topics.

Demystifying the pathway of assessment and treatment for bipolar disorder - utilising co-production and algorithms to personalise the approach

Jessica Nicholls-Mindlin ${ }^{1 *}$, Angus McLellan², David Gee², Lauren Fuzi ${ }^{2}$, Matthew Taylor ${ }^{3}$ and Digby Quested ${ }^{3}$

${ }^{1}$ University of Oxford; ${ }^{2}$ Oxford Health NHS Foundation Trust and

${ }^{3}$ Oxford Health NHS Foundation Trust, Department of Psychiatry,

University of Oxford

${ }^{\star}$ Corresponding author.

doi: 10.1192/bjo.2021.420

Aims. To develop an evidence based, patient centred treatment pathway for people experiencing symptoms of bipolar disorder (BD), modifiable to include local resources.

Method. This project was developed in line with current approaches to service development such as coproduction, with patient and public involvement (PPI) and enhancing personalisation of treatment in medicine. As part of a local initiative, a multidisciplinary team was brought together to understand and analyse the current local pathway for those affected by BD. It was found 